\title{
Spectral line broadening in quantum wells due to Coulomb interaction of current carriers
}

\author{
A.A. Afonenko ${ }^{\mathrm{a}, *}$, V.K. Kononenko ${ }^{\mathrm{b}}$ \\ ${ }^{a}$ Quantum Radiophysics and Electronics, Belarussian State University, 4, Fr. Skorini av, 220050 Minsk, Belarus \\ ${ }^{\mathrm{b}}$ Stepanov Institute of Physics, NASB, 220072 Minsk, Belarus
}

Received 6 March 2005; received in revised form 30 May 2005; accepted 19 June 2005

Available online 1 August 2005

\begin{abstract}
A theoretical analysis of emission line broadening due to Coulomb interaction of carriers is performed. An analytical approximation for the spectral line shape function with exponential decays is derived by using the perturbation theory for many-body electron-hole systems for both non-degenerate and degenerate conditions. An explanation of the experimentally observed spectral line asymmetry and the linewidth change as a function of the temperature and the excitation level is given.
\end{abstract}

(C) 2005 Elsevier B.V. All rights reserved.

PACS: 78.55.-m; 78.67.-n

Keywords: Quantum-well heterostructure; Optical transitions; Line broadening; Coulomb interaction

\section{Introduction}

An adequate theoretical description of the spectral characteristics of semiconductor structures requires the contribution of emission line broadening effects to be considered. The analysis of emission processes within the framework of the density matrix formalism with the empirical interband polarization decay time as well as the

\footnotetext{
*Corresponding author. Tel./fax: + 375172121016 .

E-mail addresses: afonenko@bsu.by (A.A. Afonenko), lavik@dragon.bas-net.by (V.K. Kononenko).
}

description of many-body processes by the firstorder perturbation theory with the energy-independent carrier relaxation time result in the Lorentzian shape of the homogeneously broadened emission line [1]. The examination of laser structures being perfect in the crystallographic respect, in which the effect of spatial inhomogeneity on the spectral broadening has been reduced to a negligible level, has revealed that the line of the homogeneous broadening has exponential tails $[2,3]$. It was found that the logarithmic decrement of decay and the linewidth increase with the temperature and injection current growth. The 
evident asymmetry of the emission line profile was observed at $4.2 \mathrm{~K}$.

Theoretical substantiations of the non-Lorentzian shape of the emission line are obtained by taking into account the non-Markovian processes [4-7]. The emission processes kinetics is described by the density-matrix formalism. Non-Markovian relaxation behavior was ensured by the autocorrelation function of the interaction Hamiltonian in the equation for the off-diagonal element of the density matrix. The time-dependent damping factors of the dipole momentum of electron-hole pairs due to carrier-carrier and carrier-LO phonon scatterings were derived in $[4,5]$.

A comprehensive quantum-mechanical description of spontaneous emission, based on coupled semiconductor luminescence and Bloch equations, is presented in [8-10]. The solution of the resulting eigenvalue/eigenvectors problem allowed to take into account the coupling of the polarization for various $\mathbf{k}$-states and to describe excitonic emission properties of quantum-well (QW) heterostructures. The dephasing of photon-assisted polarization due to Coulomb interaction of carriers was treated within the Markov approximation.

A rigorous formulation for the luminescence signal in terms of current-density fluctuations was developed in [11-13]. A spontaneous emission was derived by explicitly solving the Bethe-Salpeter equation. Coulomb interaction of carriers was considered in the Hartree-Fock approximation.

Treating the Coulomb interaction within the Markov limit allows to carry out the correct calculation of the shape and the spectrum maximum position. However, to be able to simulate the spectral tails correctly, the non-Markovian relaxation behavior has to be taken into account. The study of emission processes in the suggested models can be performed only numerically. It makes it difficult, therefore, to analyze the influence of excitation conditions and parameters of semiconductor materials on the spectrum profile.

In the present paper, the influence of Coulomb interaction of carriers on the emission line shape in QWs is studied. Similar to the analysis $[4,5,8]$, the dephasing processes are taken into account in quadratic order in the Coulomb interaction, but additional terms are considered that result in the coupling of the radiative transition with various $\mathbf{k}$-states. The basic idea of the method consists in the determination of the functional dependence of tails of the emission line function by means of the perturbation theory [14]. Then the spectral line function is extrapolated into its central part by using the normalization requirement. Here the degeneracy in the statistical distribution and spin of particles are taken into consideration. An analytical shape function for the homogeneously broadened spectral line is obtained in the parabolic band approximation. The asymmetry of the spectral line and the linewidth dependence on the temperature and the excitation level are analyzed.

\section{Probability of optical transitions in view of carriers Coulomb interaction}

Let us consider spontaneous optical transitions, where a state with $n$-electron-hole pairs changes to a state with $(n-1)$-electron-hole pairs. We have chosen single-particle wave functions in the form of Bloch functions with various wave vectors. Different second-quantization operators are used for the states in the conduction and valence bands. The electromagnetic-interaction operator, which describes radiative transitions with conservation of the wave vector, can be written in the form

$\hat{V}_{\mathrm{ph}} \sim \sum_{r, r^{\prime}} R_{r^{\prime} r} \hat{b}_{r^{\prime}} \hat{a}_{r}$,

where $\hat{a}_{r}$ and $\hat{b}_{r}$ are the annihilation operators for a conduction electron in an $r$-state, which are characterized by the wave vector $\mathbf{k}_{r}$ and spin $s_{r}$.

$R_{r^{\prime} r}=\delta_{-\mathbf{k}_{r^{\prime}}, \mathbf{k}_{r}} \delta_{-s_{r^{\prime}}, s_{r}}$

represents the wave vector and the spin conservation at the optical transitions. In expression (1) the creation operators for photons $\hat{c}_{r}^{\dagger}$ are omitted, because in further analysis only the transitions are considered, when there are no photons in the initial state and one photon is created after recombination of the electron and the hole, 
i.e. non-zero matrix elements are $\left\langle 1\left|\hat{c}_{r}^{\dagger}\right| 0\right\rangle=1$. The momentum matrix elements are supposed to be independent from the wave vectors of the electron states and photons, involved in the transition. The polarization characteristics are not considered.

We introduce the interaction between conduction electrons, between holes, and between electrons and holes into the Coulomb interaction operator, disregarding exchange interaction between electrons and holes and interaction resulting in processes of interband transfer, i.e.,

$$
\begin{aligned}
\hat{V}= & \sum_{i, j, i^{\prime}, j^{\prime}} Q_{i^{\prime} j^{\prime} j i} V_{\mathbf{k}_{i}-\mathbf{k}_{i^{\prime}}}\left(\frac{1}{2} \hat{a}_{i^{\prime}}^{\dagger} \hat{j}_{j^{\prime}}^{\dagger} \hat{a}_{j} \hat{a}_{i}\right. \\
& \left.+\frac{1}{2} \hat{b}_{i^{\prime}}^{\dagger} \hat{b}_{j^{\prime}}^{\dagger} \hat{b}_{j} b_{i}-\hat{a}_{i^{\prime}}^{\dagger} \hat{b}_{j^{\prime}}^{\dagger} \hat{b}_{j} \hat{a}_{i}\right) .
\end{aligned}
$$

Here, $\hat{a}^{\dagger}$ and $\hat{b}^{\dagger}$ are the creation operators for a conduction band electron and a hole, respectively, with a certain wave vector

$Q_{i^{\prime} j^{\prime} j i}=\delta_{\mathbf{k}_{i^{\prime}}+\mathbf{k}_{j^{\prime}}, \mathbf{k}_{j}+\mathbf{k}_{i}} \delta_{s_{i^{\prime}}, S_{i}} \delta_{s_{j^{\prime}}, S_{j}}$

represents the momentum and the spin conservation, $V_{\mathbf{k}_{i}-\mathbf{k}_{i^{\prime}}}$ is the matrix element of the Coulomb interaction.

The operator that introduces the first-order perturbation-theory corrections into the initial many-body state can be written formally as

$$
\begin{aligned}
\hat{\Psi}_{c v}= & +\sum_{i, j, i^{\prime}, j^{\prime}} Q_{i^{\prime} j^{\prime} j i}\left[\frac{\hat{a}_{i^{\prime}}^{\dagger} \hat{a}_{j^{\prime}}^{\dagger} \hat{a}_{j} \hat{a}_{i}}{2\left(E_{c i}+E_{c j}-E_{c j^{\prime}}-E_{c i^{\prime}}\right)}\right. \\
& +\frac{\hat{b}_{i^{\prime}}^{\dagger} \hat{j}_{j^{\prime}}^{\dagger} \hat{b}_{j} \hat{b}_{i}}{2\left(E_{v i}+E_{v j}-E_{v j^{\prime}}-E_{v i^{\prime}}\right)} \\
& \left.-\frac{\hat{a}_{i^{\prime}}^{\dagger} \hat{b}_{j^{\prime}}^{\dagger} \hat{b}_{j} \hat{a}_{i}}{E_{c i}+E_{v j}-E_{v j^{\prime}}-E_{c i^{\prime}}}\right],
\end{aligned}
$$

where $E_{c}$ and $E_{v}$ are the energies of one-body states of the conduction and valence bands counted from the energies of the ground one-body states with $\mathbf{k}=0$. An ambiguity of the expansion coefficients of terms with similar energies of initial and mixed states will be considered separately in further analysis.
Expression (5) is used to find the first-order Coulomb corrections to the radiative transitions. For the conduction band, we can write

$$
\begin{aligned}
\hat{M}_{c c}= & \sum_{r, r^{\prime}} \sum_{i, j, j^{\prime}} R_{r^{\prime} r} Q_{r j^{\prime} j i} V_{\mathbf{k}_{i}-\mathbf{k}_{r}} \\
& \times \frac{\hat{b}_{r^{\prime}} \hat{a}_{j^{\prime}}^{\dagger} \hat{a}_{j} \hat{a}_{i}}{E_{c i}+E_{c j}-E_{c j^{\prime}}-E_{c r}} .
\end{aligned}
$$

A similar expression is valid for the valence band. The operator of the first-order corrections for Coulomb interaction between carriers in different bands to the radiative transitions has the form

$$
\begin{aligned}
\hat{M}_{c v}= & -\sum_{r, r^{\prime}} \sum_{i, j, i^{\prime} \neq r} R_{r^{\prime} r} Q_{i^{\prime} \prime r i j} V_{\mathbf{k}_{i}-\mathbf{k}_{i^{\prime}}} \\
& \times \frac{\hat{a}_{r} \hat{a}_{i^{\prime}}^{\dagger} \hat{b}_{j} \hat{a}_{i}}{E_{c i}+E_{v j}-E_{v r^{\prime}}-E_{c i^{\prime}}} \\
& -\sum_{r, r^{\prime}} \sum_{i, j, j j^{\prime} \neq r^{\prime}} R_{r^{\prime} r} Q_{r j^{\prime} j i} V_{\mathbf{k}_{i}-\mathbf{k}_{r}} \\
& \times \frac{\hat{b}_{r^{\prime}} \hat{b}_{j^{\prime}}^{\dagger} \hat{b}_{j} \hat{a}_{i}}{E_{c i}+E_{v j}-E_{v j^{\prime}}-E_{c r}} \\
& -\sum_{r, r^{\prime}} \sum_{i, j} R_{r^{\prime} r} Q_{r r^{\prime} j i} V_{\mathbf{k}_{i}-\mathbf{k}_{r}} \\
& \times \frac{\hat{b}_{j} \hat{a}_{i}\left(1-n_{c r}-n_{v r^{\prime}}\right)}{E_{c i}+E_{v j}-E_{v r^{\prime}}-E_{c r}} .
\end{aligned}
$$

Operator (6) and the first term in (7) describe the recombination of an electron and a hole with transfer of excess momentum to another electron. The third term in (7) describes the variation in the recombination probability for an electron-hole pair with a zero quasi-momentum.

With regard to operators (6) and (7), the normalized probability of a radiative transition with energy $\hbar \omega$, which describes spontaneous transitions from the $n$-electron state (this state, among others, includes the one-particle states of the conduction band with the wave vectors $\mathbf{k}_{1}, \mathbf{k}_{2}$ and the one-particle state of the valence band with the wave vector $\left.\mathbf{k}_{3}\right)$ to the $(n-1)$-electron state (which, instead of the aforementioned set of oneparticle states, includes only the one-electron state of the conduction band with the wave vector $\mathbf{k}_{4}$ ) 
has the form

$$
\begin{aligned}
W_{c}= & \left(\frac{V_{\mathbf{k}_{1}+\mathbf{k}_{3}}-V_{\mathbf{k}_{2}+\mathbf{k}_{3}}}{E_{c \mathbf{k}_{1}}+E_{c \mathbf{k}_{2}}-E_{c \mathbf{k}_{4}}-E_{c-\mathbf{k}_{3}}}\right. \\
& -\frac{V_{\mathbf{k}_{1}+\mathbf{k}_{3}}}{E_{c \mathbf{k}_{2}}+E_{v \mathbf{k}_{3}}-E_{v-\mathbf{k}_{1}}-E_{c \mathbf{k}_{4}}} \\
& \left.+\frac{V_{\mathbf{k}_{2}+\mathbf{k}_{3}}}{E_{c \mathbf{k}_{1}}+E_{v \mathbf{k}_{3}}-E_{v-\mathbf{k}_{2}}-E_{c \mathbf{k}_{4}}}\right)^{2} \\
& \times \delta\left(E_{c \mathbf{k}_{1}}+E_{c \mathbf{k}_{2}}+E_{v \mathbf{k}_{3}}-E_{c \mathbf{k}_{4}}-\Delta \hbar \omega\right),
\end{aligned}
$$

where $\Delta \hbar \omega=\hbar \omega-E_{\mathrm{g}}, E_{\mathrm{g}}$ is the effective band gap in the QW.

If the states in the conduction band with wave vectors $\mathbf{k}_{1}, \mathbf{k}_{2}$ have different spins, then the corresponding transition probability can be obtained from expression (8), where matrix element $V_{\mathbf{k}_{2}+\mathbf{k}_{3}}$ or $V_{\mathbf{k}_{1}+\mathbf{k}_{3}}$ equals zero. For direct transitions with $\mathbf{k}$, the normalized probability is just

$$
W_{d}=\delta\left(E_{c \mathbf{k}}+E_{v-\mathbf{k}}-\Delta \hbar \omega\right) .
$$

The probability of radiative transition (8) is an analogue of the Fourier transform of the damping factor $[4,5]$. The products of the terms with different poles describe the coupling of the radiative transition with various $\mathbf{k}$-states due to the Coulomb interaction of carriers, and they are not presented in analysis [4,5]. Such terms would appear in model [8], if the full equation of motion is analyzed for expectation values of four carriers and one-photon operators instead of treating only the Coulomb part of the Hamiltonian. The coupling terms can play an important role. For example, instead of interband radiative transitions if we consider the intersubband radiative transitions between the subbands with the equal curvature (that can be taken into account in (8) by the replacement $E_{v \mathbf{k}} \rightarrow-E_{c-\mathbf{k}}$ ), then the probability $W_{c}$ becomes zero. Therefore the line width at intersubband transitions becomes strongly dependent on both the subbands massdispersion and QW width [15].

In expression (8) we used the energies of the initial and final many-electron states, with the Coulomb interaction being disregarded. If the Coulomb interaction even in the first-order perturbation theory is included in the analysis, $W_{c}$ becomes a function of all the one-electron components of the many-particle state, which significantly complicates further analysis. We can conclude qualitatively that the change in the energy of an ensemble of particles due to their interaction should shift the argument of the delta function to lower energies (the effect of band gap shrinkage) and broaden the delta function itself upon averaging over the set of many-electron states. The latter circumstance also leads to the broadening of emission lines. The effect of this mechanism requires separate investigation and is beyond the scope of this study.

When the argument of the delta function is taken into account, one can see that, for specified different values of $\mathbf{k}_{1}, \mathbf{k}_{2}, \mathbf{k}_{3}$ the value of $W_{c}$, depending on the energy of emitted photons, has three resonance peaks near the energies of the direct transitions with the corresponding wave vectors. This phenomenon is due to the fact that the perturbation theory approach was used; it would be absent if an exact basis expansion of many-electron states had been used instead of approximate expression (5). Far from the resonances, only the basis components of expansion (5) with small coefficients are presented in transition probability (8), which justifies the application of the perturbation theory in these spectral regions.

To derive the line shape function it is necessary to separate in expression (8) the terms with the poles corresponding to different wave vectors $\mathbf{k}_{1}$, $\mathbf{k}_{2}, \mathbf{k}_{3}$, i.e.,

$$
\begin{aligned}
W_{c}= & W_{c 1}\left(\Delta \hbar \omega-E_{r \mathbf{k}_{1}}\right)+W_{c 2}\left(\Delta \hbar \omega-E_{r \mathbf{k}_{2}}\right) \\
& +W_{c 3}\left(\Delta \hbar \omega-E_{r-\mathbf{k}_{3}}\right)
\end{aligned}
$$

where

$$
\begin{aligned}
W_{c 3}\left(\Delta \hbar \omega-E_{r-\mathbf{k}_{3}}\right) & {\left[\frac{V_{\mathbf{k}_{1}+\mathbf{k}_{3}}\left(V_{\mathbf{k}_{1}+\mathbf{k}_{3}}-V_{\mathbf{k}_{2}+\mathbf{k}_{3}}\right)\left(E_{r-\mathbf{k}_{3}}-E_{r \mathbf{k}_{1}}\right)^{2}}{\left(\Delta \hbar \omega-E_{r-\mathbf{k}_{3}}\right)^{2}+\left(\Delta \hbar \omega-E_{r \mathbf{k}_{1}}\right)^{2}}\right.} \\
& \left.+\frac{V_{\mathbf{k}_{2}+\mathbf{k}_{3}}\left(V_{\mathbf{k}_{2}+\mathbf{k}_{3}}-V_{\mathbf{k}_{1}+\mathbf{k}_{3}}\right)\left(E_{r-\mathbf{k}_{3}}-E_{r \mathbf{k}_{2}}\right)^{2}}{\left(\Delta \hbar \omega-E_{r-\mathbf{k}_{3}}\right)^{2}+\left(\Delta \hbar \omega-E_{r \mathbf{k}_{2}}\right)^{2}}\right] \\
& \times \frac{\delta\left(E_{c \mathbf{k}_{1}}+E_{c \mathbf{k}_{2}}+E_{v \mathbf{k}_{3}}-E_{c \mathbf{k}_{4}}-\Delta \hbar \omega\right)}{\left(\Delta \hbar \omega-E_{r-\mathbf{k}_{3}}\right)^{2}},
\end{aligned}
$$




$$
\begin{aligned}
W_{c 1}\left(\Delta \hbar \omega-E_{r-\mathbf{k}_{1}}\right) & \\
= & {\left[\frac{V_{\mathbf{k}_{1}+\mathbf{k}_{3}}\left(V_{\mathbf{k}_{1}+\mathbf{k}_{3}}-V_{\mathbf{k}_{2}+\mathbf{k}_{3}}\right)\left(E_{r-\mathbf{k}_{3}}-E_{r \mathbf{k}_{1}}\right)^{2}}{\left(\Delta \hbar \omega-E_{r-\mathbf{k}_{3}}\right)^{2}+\left(\Delta \hbar \omega-E_{r \mathbf{k}_{1}}\right)^{2}}\right.} \\
& \left.+\frac{V_{\mathbf{k}_{1}+\mathbf{k}_{3}} V_{\mathbf{k}_{2}+\mathbf{k}_{3}}\left(E_{r \mathbf{k}_{1}}-E_{r \mathbf{k}_{2}}\right)^{2}}{\left(\Delta \hbar \omega-E_{r \mathbf{k}_{1}}\right)^{2}+\left(\Delta \hbar \omega-E_{r \mathbf{k}_{2}}\right)^{2}}\right] \\
& \times \frac{\delta\left(E_{c \mathbf{k}_{1}}+E_{c \mathbf{k}_{2}}+E_{v \mathbf{k}_{3}}-E_{c \mathbf{k}_{4}}-\Delta \hbar \omega\right)}{\left(\Delta \hbar \omega-E_{r \mathbf{k}_{1}}\right)^{2}} .
\end{aligned}
$$

The probability $W_{c 2}\left(\Delta \hbar \omega-E_{r \mathbf{k}_{2}}\right)$ is obtained from expression (12) for $W_{c 1}\left(\Delta \hbar \omega-E_{r \mathbf{k}_{1}}\right)$ by the interchange $\mathbf{k}_{1} \leftrightarrow \mathbf{k}_{2}$.

\section{Statistical averaging of the optical transitions probability}

Quantum-mechanical probability (8) with the specified values of $\mathbf{k}_{1}, \mathbf{k}_{2}, \mathbf{k}_{3}$, and $\mathbf{k}_{4}$ can be applied to the entire ensemble of many-particle states. In order to calculate the resulting contribution to the rate of spontaneous transitions, it is necessary to sum expression (8) over all the initial manyparticle states containing the noted one-particle state, with regard to their occupation numbers. Summation over the final states is left out in accordance with the quasi-momentum conservation law and the existence of only one final state with $\mathbf{k}_{4}=\mathbf{k}_{1}+\mathbf{k}_{2}+\mathbf{k}_{3}$. The calculation is performed by disregarding the Coulomb interaction, as for the delta function in expression (8).

Using thermodynamic filling factors

$$
\begin{aligned}
& f_{c}\left(E_{c \mathbf{k}}\right)=\left(1+\exp \left[\left(E_{\mathrm{g}}+E_{c \mathbf{k}}-F_{\mathrm{e}}\right) / k T\right]\right)^{-1}, \\
& f_{v}\left(E_{v \mathbf{k}}\right)=\left(1+\exp \left[\left(F_{\mathrm{h}}+E_{v \mathbf{k}}\right) / k T\right]\right)^{-1},
\end{aligned}
$$

we found the four-state statistical factor, i.e.,

$p_{\mathbf{k}_{1}, \mathbf{k}_{2}, \mathbf{k}_{3}}=f_{c 1} f_{c 2} f_{v 3}\left(1-f_{c 4}\right)$.

Assuming electroneutrality of the QW layer in the approximation of nondegenerate electron gas we obtained

$$
\begin{aligned}
p_{\mathbf{k}_{1}, \mathbf{k}_{2}, \mathbf{k}_{3}}= & \sqrt{\frac{N_{v}}{N_{c}}} \exp \left(\frac{3\left(\Delta F-E_{\mathrm{g}}\right)}{2 k T}\right. \\
& \left.-\frac{E_{c \mathbf{k}_{1}}+E_{c \mathbf{k}_{2}}+E_{v \mathbf{k}_{3}}}{k T}\right),
\end{aligned}
$$

where $N_{c}$ and $N_{v}$ are the effective states density for electrons and holes, $\Delta F=F_{\mathrm{e}}-F_{\mathrm{h}}$ is the difference of the quasi-Fermi levels for electrons $F_{\mathrm{e}}$ and holes $F_{\mathrm{h}}$.

In order to obtain contributions to the spectral broadening of individual components of the optical transitions probability, one has to fix the wave vector responsible for a pole and integrate (with regard to the statistical factor) $W_{\text {ci }}$ over the phase space of the remaining wave vectors. Finally, denoting the argument of the obtained functions by the same variable $\mathbf{k}$ and summing these functions, we found the line shape for the direct transitions with the indicated wave vector. The result is valid for the entire spectral range (except for the region $\Delta \hbar \omega-E_{r \mathbf{k}} \approx 0$ ). The values of the broadening function in this region can be estimated by way of its restriction and normalization to the non-perturbed probability of direct optical transitions (with the Coulomb interaction not taken into account).

\section{Calculation of the emission spectral line}

In order to calculate the line shape function, it is necessary to specify the dependence of the energies of one-electron states on the wave vector. In the parabolic band approximation, for electron and hole energies we have

$E_{c \mathbf{k}}=\frac{\hbar^{2} \mathbf{k}^{2}}{2 m_{c}}, \quad E_{v \mathbf{k}}=\frac{\hbar^{2} \mathbf{k}^{2}}{2 m_{v}}$,

where $m_{c}$ and $m_{v}$ are the electron and hole effective masses, respectively. We mention here that in the paper we use positive energies $E_{c \mathbf{k}}$ and $E_{v \mathbf{k}}$. The band gap energy $E_{\mathrm{g}}$ and signs of one-particle energies are explicitly treated in expressions (13) and (15). This make it possible to obtain the result for the process of photon emission with participation of two holes and one electron by a simple replacement of indices: $c \leftrightarrow v$. 
We use the matrix element of the Coulomb interaction in a two-dimensional system in the simplest form

$V_{\Delta \mathbf{k}}=\frac{e^{2}}{2 \varepsilon \varepsilon_{0}|\Delta \mathbf{k}| S}$,

where $\varepsilon$ is the dielectric constant of the semiconductor material, and $S$ is the area of the QW layer. The screening effects are not important here because at $\mathbf{k}=0$ probabilities (12) and (11) also have poles and the resulting singularities are eliminated in a semi-empirical manner.

Let us consider a component of the probability of optical transitions (11) that has a pole when the energy of light photons equals the energy of direct transitions involving the states of the valence band with wave vector $\mathbf{k}_{3}: \Delta \hbar \omega=E_{r-\mathbf{k}_{3}}$. To obtain the line shape function, we integrate the product of (11) and (15) over the phase space of wave vectors $\mathbf{k}_{1}$ and $\mathbf{k}_{2}$,

$$
\begin{aligned}
& L_{c 3}(\Delta \hbar \omega) \\
& =\left\{\begin{array}{l}
\frac{1}{2} \\
1
\end{array} \int p_{\mathbf{k}_{1}, \mathbf{k}_{2}, \mathbf{k}_{3}} \cdot W_{c 3}\left(\Delta \hbar \omega-E_{r-\mathbf{k}_{3}}\right) \frac{S d \mathbf{k}_{1}}{(2 \pi)^{2}} \frac{S d \mathbf{k}_{2}}{(2 \pi)^{2}} .\right.
\end{aligned}
$$

Here, the upper multiplier within the curly bracket corresponds to interband transitions, involving the electrons with the same spins, and the lower-with different ones. The factor $\frac{1}{2}$ takes into account the indistinguishability of many-body states at permutations of $\mathbf{k}_{1}$ and $\mathbf{k}_{2}$.

Integral (18) cannot be found analytically, but it can be estimated for limiting cases $\left|\Delta \hbar \omega^{\prime}\right| \gg k T$ and $\left|\Delta \hbar \omega^{\prime}\right| \gg E_{r-\mathbf{k}_{3}}$, where $\Delta \hbar \omega^{\prime}=\Delta \hbar \omega-E_{r}$. Upon integration, it is convenient to perform a linear transformation of integration variables that reduces a quadratic form of projections of wave vectors in the delta function to a diagonal representation. For the long-wavelength tail of the spectral line $\left(\Delta \hbar \omega^{\prime}<0\right)$, we obtain

$$
\begin{aligned}
& L_{c 3}^{-}\left(\Delta \hbar \omega^{\prime}\right) \\
& =A\left\{\begin{array}{l}
\frac{m_{c}^{2}\left(m_{c}^{2}-4 m_{c} m_{r}-24 m_{r}^{2}\right)}{2\left(m_{c}^{2}+4 m_{c} m_{r}+8 m_{r}^{2}\right)^{2}} \frac{(k T)^{2}}{\left(\Delta \hbar \omega^{\prime}\right)^{4}} \\
\frac{m_{c}^{2}}{\left(m_{c}^{2}+4 m_{c} m_{r}+8 m_{r}^{2}\right)} \frac{k T}{\left|\Delta \hbar \omega^{\prime}\right|^{3}}
\end{array}\right.
\end{aligned}
$$

$$
\times\left\{\begin{array}{l}
I_{0}\left(\sqrt{\left.8 \frac{m_{r}}{m_{c}} \frac{\left|\Delta \hbar \omega^{\prime}\right| E_{r}}{(k T)^{2}}\right) \exp \left(\frac{3\left(\Delta F-E_{\mathrm{g}}\right)}{2 k T}\right.}\right. \\
\left.-\frac{E_{r}}{k T}\left(1+\frac{m_{r}}{m_{c}}\right)+\frac{\Delta \hbar \omega^{\prime}}{k T}\right) \\
\sqrt{\frac{m_{c}}{m_{v}}} f_{c}^{2}\left(-\frac{\Delta \hbar \omega^{\prime}}{2 k T}\right) f_{v}(0) .
\end{array}\right.
$$

Here, the terms in the first curly bracket correspond to the different spin combinations of interacting particles (with identical or non-identical spins); in the second curly brackets the upper and lower factors correspond to the nondegenerated and degenerated (at $E_{r}=0$ ) cases, and $I_{0}$ is the modified Bessel function of the first kind, $m_{r}^{-1}=m_{c}^{-1}+m_{v}^{-1}$ :

$A=\left(\frac{e^{2}}{2 \varepsilon \varepsilon_{0}}\right)^{2} \frac{\sqrt{m_{c} m_{v}}}{8 \pi^{2} \hbar^{2}}=\operatorname{Ry} \frac{\sqrt{m_{c} m_{v}}}{m_{e} \varepsilon^{2}}$,

$\mathrm{Ry} \approx 13.6 \mathrm{eV}$. For the short-wavelength tail of the spectral line $\left(\Delta \hbar \omega^{\prime}>0\right)$ we have

$$
\begin{aligned}
& L_{c 3}^{+}\left(\Delta \hbar \omega^{\prime}\right) \\
& =A\left\{\begin{array}{l}
\frac{m_{c}^{2}\left(m_{c}^{2}+4 m_{c} m_{r}-24 m_{r}^{2}\right)}{2\left(m_{c}^{2}-4 m_{c} m_{r}+8 m_{r}^{2}\right)^{2}} \frac{(k T)^{2}}{\left(\Delta \hbar \omega^{\prime}\right)^{4}} \\
\frac{m_{c}^{2}}{\left(m_{c}^{2}-4 m_{c} m_{r}+8 m_{r}^{2}\right)} \frac{k T}{\left(\Delta \hbar \omega^{\prime}\right)^{3}}
\end{array}\right. \\
& \times\left\{\begin{array}{l}
\exp \left(\frac{3\left(\Delta F-E_{\mathrm{g}}\right)}{2 k T}-\frac{E_{r}}{k T}-\frac{\Delta \hbar \omega^{\prime}}{k T}\right), \\
\sqrt{\frac{m_{c}}{m_{v}}} f_{c}^{2}\left(\frac{\Delta \hbar \omega^{\prime}}{2 k T}\right) f_{v}(0) .
\end{array}\right.
\end{aligned}
$$

Terms of expressions (19) and (20) for transitions with participation of electrons with identical spins are not positive at definite ratios of the effective masses. This is because (11) includes the contribution related to coupling of the radiative transition with various k-states. Since the probability of spontaneous transitions (8) cannot be negative, in the resulting rate of spontaneous transitions at an arbitrary frequency, the negative contribution of expressions (19) and (20) obtained for small values of $E_{r-\mathbf{k}_{3}}$ should be compensated by the contribution at large values or the remaining transition probability components (12). 
By analogy to the previous case for the component of the spectral-line profile corresponding to the probability of optical transitions (12), we have the following expressions for the longwavelength tail of the spectral line $\left(\Delta \hbar \omega^{\prime}<0\right)$ :

$$
\begin{aligned}
& L_{c 1}^{-}\left(\Delta \hbar \omega^{\prime}\right) \\
&=\frac{A}{5} \frac{k T}{\left|\Delta \hbar \omega^{\prime}\right|^{3}} \\
& \times\left\{\begin{array}{l}
\frac{m_{c}\left(m_{c}^{3}+4 m_{c}^{2} m_{v}-2 m_{c} m_{v}^{2}+2 m_{v}^{3}\right)}{2\left(m_{c}^{4}+2 m_{c}^{2} m_{v}^{2}+2 m_{v}^{4}\right)} \\
1 \\
\times \\
\left.\quad-\frac{E_{r}}{k T}\left(1+\frac{m_{r} m_{c}}{m_{v}^{2}}\right)+\frac{m_{c}}{m_{v}} \frac{\Delta \hbar \omega^{\prime}}{k T}\right), \\
\sqrt{\frac{m_{c}}{m_{v}}} f_{c}(0) f_{c}\left(-\frac{m_{c} m_{r}}{m_{v}^{2}} \frac{\Delta \hbar \omega}{k T}\right) \\
\times f_{v}\left(-\frac{m_{r}}{m_{v}} \frac{\Delta \hbar \omega}{k T}\right),
\end{array}\right.
\end{aligned}
$$

and for the short-wavelength tail of the line $\left(\Delta \hbar \omega^{\prime}>0\right)$ :

$$
\begin{aligned}
L_{c 1}^{+}\left(\Delta \hbar \omega^{\prime}\right)= & A \frac{k T}{\left(\Delta \hbar \omega^{\prime}\right)^{3}}\left\{\begin{array}{l}
\frac{1}{2} \\
1
\end{array}\right. \\
& \times\left\{\begin{array}{l}
\exp \left(\frac{3\left(\Delta F-E_{\mathrm{g}}\right)}{2 k T}-\frac{E_{r}}{k T}-\frac{\Delta \hbar \omega^{\prime}}{k T}\right), \\
\sqrt{\frac{m_{c}}{m_{v}}} f_{c}(0) f_{c}\left(\frac{m_{r}}{m_{c}} \frac{\Delta \hbar \omega}{k T}\right) f_{v}\left(\frac{m_{r}}{m_{v}} \frac{\Delta \hbar \omega}{k T}\right) .
\end{array}\right.
\end{aligned}
$$

Expressions (21) and (22) are positively defined at any ratio of the effective masses and, in comparison with expressions (19) and (20), show a slower decay, which ensures positiveness of the tails of the resulting line shape function.

As the numerical calculations show, the approximations obtained for components of the spectral line yield a sufficiently good approach where detuning $\Delta \hbar \omega^{\prime}>k T$ (Fig. 1). For the emission transitions with $E_{r}<k T$, the dominating contribution to the broadening at the long-wavelength spectrum side $\left(\left|\Delta \hbar \omega^{\prime}\right| \gg k T\right)$ is given by the recombination in electron-hole pairs with transfer of the excess momentum to another electron (upon the electron-hole Coulomb interaction), $L_{c 1}\left(\Delta \hbar \omega^{\prime}\right)$, and in the short-wavelength part of the spectrum $\left(\Delta \hbar \omega^{\prime} \gg k T\right)$ - with transfer of the excess momentum either to an electron or to a hole: $L_{c 1}\left(\Delta \hbar \omega^{\prime}\right)$ and $L_{v 1}\left(\Delta \hbar \omega^{\prime}\right)$, respectively.

\section{Extrapolation of the emission line shape function near the resonance}

Exponential and power factors are clearly distinguished in expressions (19)-(22); these factors originate from the statistical and quantummechanical properties of a many-body system. The exponent of the exponential multiplier, which is due to statistical factor (15), includes the minimum sum of the energies of interacting carriers at which the difference between the energies of the initial and final many-particle states (with regard to the quasi-momentum conservation) corresponds to the specified energy detuning from the center of the emission line, $\Delta \hbar \omega^{\prime}$. In the other multiplier, the part with the exponent of 2 is due to the use of perturbation theory, while the remaining part is related to the form of matrix element of the Coulomb interaction (17). Thus, the spectral-line tails calculated by the above method would formally coincide with the Lorentzian function if the matrix element was assumed to be constant and the quasi-momentum conservation law was disregarded.

Therefore, we perform restriction of the spectral line in the region of small detunings by analogy with the Lorentzian shape and matching of the exponential factors for positive and negative detunings as in Ref. [3]. Assuming that the total intensity of the emission line is the same as in the one-electron approximation for direct radiative transitions, we write the form factor of homogeneous broadening for the nondegenerate case 

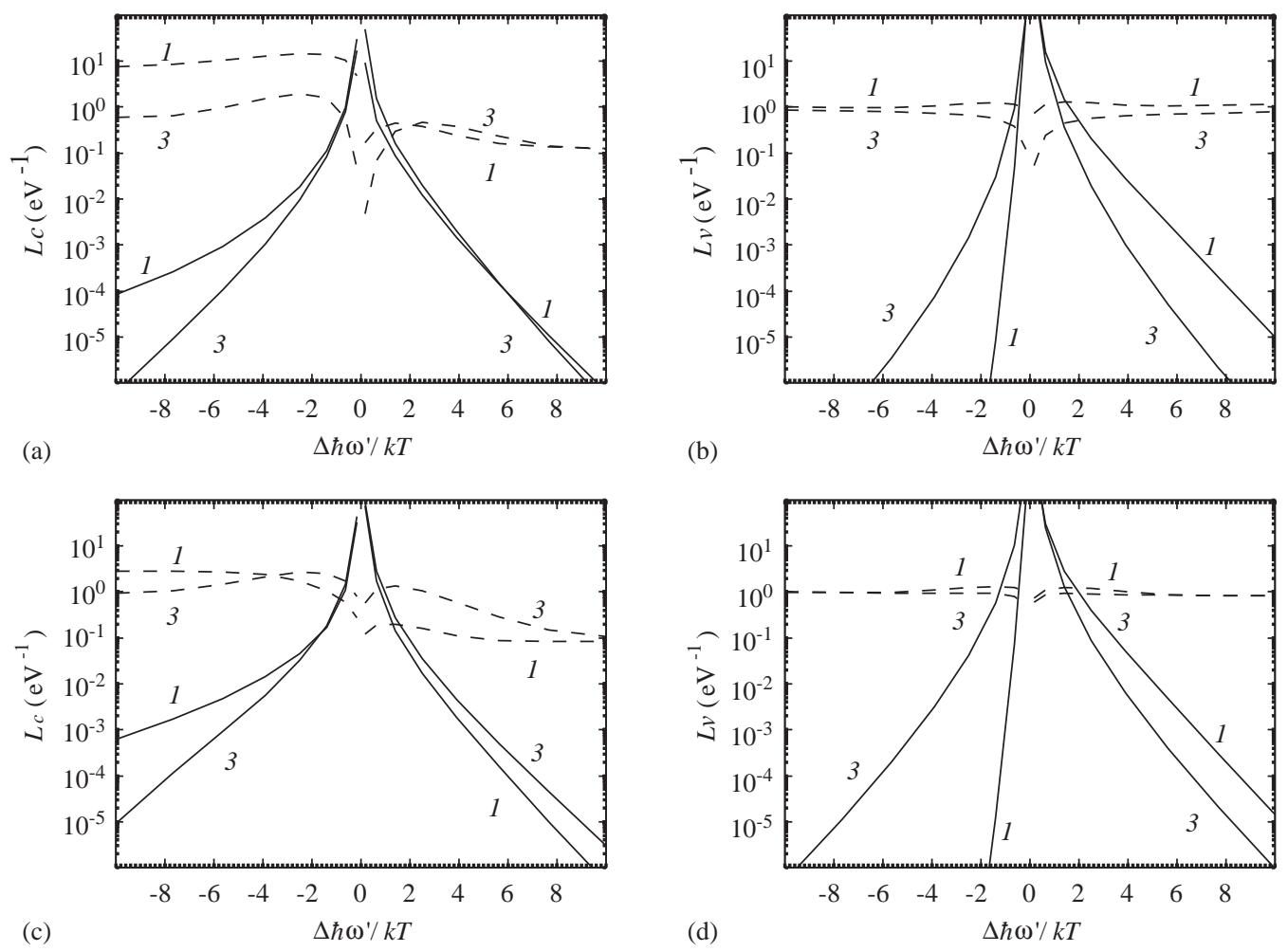

Fig. 1. Components of the broadened spectral line corresponding to the electron-hole pair recombination processes with transfer of the excess momentum to (a), (c) an electron $L_{c}$ and (b), (d) to a hole $L_{v}$ with the same (a), (b) and the opposite (c), (d) spins. The components of the line due to the Coulomb interaction with transfer of the excess momentum to the hole are obtained from expressions (19)-(22) by interchange $m_{c} \leftrightarrow m_{v}$. The dotted lines show the ratio of numerical integration results to approximations. Numbers 1 mark curves corresponding to processes of the interband Coulomb interaction and its coupling with the intraband Coulomb interaction processes, 3 - to processes of the intraband Coulomb interaction and its coupling with the interband Coulomb interaction processes. For line $L_{c 3}$, absolute values are plotted. $T=286 \mathrm{~K}, m_{c}=0.055 m_{e}, m_{v}=0.49 m_{e}, E_{r}=0, \Delta F-E_{\mathrm{g}}=3 \mathrm{kT}$.

as follows:

$F\left(\Delta \hbar \omega^{\prime}\right)$

$$
\approx \frac{\operatorname{Ry} \frac{\sqrt{m_{c} m_{v}}}{m_{e} \varepsilon^{2}} \exp \left(\frac{\Delta F-E_{\mathrm{g}}}{2 k T}\right) \frac{k T}{\left(\gamma^{2}+\left(\Delta h \omega^{\prime}\right)^{2}\right)^{3 / 2}}}{\left[\left(\frac{1}{5} \exp \left(\frac{m_{c}}{m_{v}} \frac{\Delta \hbar \omega^{\prime}}{k T}\right)+\frac{14}{13} \exp \left(\frac{\Delta \hbar \omega^{\prime}}{k T}\right)\right)^{-1}+\frac{5}{26} \exp \left(\frac{\Delta \hbar \omega^{\prime}}{k T}\right)\right]} .
$$

Here, we assumed that $m_{v} \gg m_{c}$ and excluded the exponential and Bessel factors, which only weakly depend on the energy of the states, from the longwavelength part on the profile. The parameter $\gamma$ is found from the normalization condition in which integral (23) over all energies of emitted photons is equal to unity. Numerical analysis of spectral line shape by direct solution of Schrödinger equation for
3-5 interacting particles [16] showed good agreement with the result obtained by the method described above. Calculations were performed for a twodimensional system with the electron-electron and hole-hole Coulomb interactions. Inclusion of the electron-hole Coulomb interaction into consideration is limited by computational difficulties.

At low excitation levels, when the emission decay near the resonance is mainly due to the power factor, the parameter $\gamma$ can be expressed analytically, i.e.,

$\gamma^{2} \approx 2.05 \cdot \operatorname{Ry} \frac{\sqrt{m_{c} m_{v}}}{m_{e} \varepsilon^{2}} k T \exp \left(\frac{\Delta F-E_{\mathrm{g}}}{2 k T}\right)$.

The decay of the spectral line near the resonance at $\gamma<k T$ is mainly due to the quantum-mechanical 
factor. At $\gamma>k T$, the statistical factor plays the dominant role. For the energies of photons $\Delta \hbar \omega^{\prime}<\gamma$, when the effect of artificial normalization is significant, the main reason for the emission-line broadening due to Coulomb interaction is the splitting of the energy levels of many-particle states.

The estimations of functional dependencies of the line in the degenerated case can be made with the following expression:

$$
\begin{aligned}
F(\Delta \hbar \omega) \approx & \operatorname{Ry} \frac{\sqrt{m_{c} m_{v}}}{m_{e} \varepsilon^{2}} \frac{G_{\mathrm{eff}}(\Delta \hbar \omega)}{f_{c}(0) f_{v}(0)} \\
& \times \frac{k T}{\left(\gamma^{2}+(\Delta \hbar \omega)^{2}\right)^{3 / 2}},
\end{aligned}
$$

where the function $G_{\text {eff }}(\Delta \hbar \omega)$ is found from the relation

$\frac{1}{G_{\text {eff }}(\Delta \hbar \omega)}=\frac{1}{G^{-}(\Delta \hbar \omega)}+\frac{1}{G^{+}(\Delta \hbar \omega)}$.

The functions $G^{-}$and $G^{+}$include statistical factors of dominant components of the emission line at long- and short-wavelength sides of the spectrum,

$$
\begin{aligned}
G^{-}(\Delta \hbar \omega)= & \frac{1}{5} \sqrt{\frac{m_{c}}{m_{v}}} f_{c}(0) f_{c}\left(-\frac{m_{c} m_{r}}{m_{v}^{2}} \frac{\Delta \hbar \omega}{k T}\right) \\
& \times f_{v}\left(-\frac{m_{r}}{m_{v}} \frac{\Delta \hbar \omega}{k T}\right) \\
& +\frac{1}{13} \sqrt{\frac{m_{c}}{m_{v}}} f_{c}^{2}\left(-\frac{\Delta \hbar \omega}{2 k T}\right) f_{v}(0) \\
& +\sqrt{\frac{m_{v}}{m_{c}}} f_{v}^{2}\left(-\frac{\Delta \hbar \omega}{2 k T}\right) f_{c}(0), \\
G^{+}(\Delta \hbar \omega)= & \frac{1}{5} \sqrt{\frac{m_{c}}{m_{v}}} f_{c}^{2}\left(\frac{\Delta \hbar \omega}{2 k T}\right) f_{v}(0) \\
& +\sqrt{\frac{m_{v}}{m_{c}}} f_{v}^{2}\left(\frac{\Delta \hbar \omega}{2 k T}\right) f_{c}(0) \\
& +2\left(\sqrt{\frac{m_{c}}{m_{v}}} f_{c}(0)+\sqrt{\frac{m_{v}}{m_{c}}} f_{v}(0)\right) \\
& \times f_{v}\left(\frac{m_{r}}{m_{v}} \frac{\Delta \hbar \omega}{k T}\right) f_{c}\left(\frac{m_{r}}{m_{c}} \frac{\Delta \hbar \omega}{k T}\right) .
\end{aligned}
$$

The non-zero energies in the Fermi-Dirac functions correspond to minimal energies of particles, one of which after Coulomb interaction (3) has

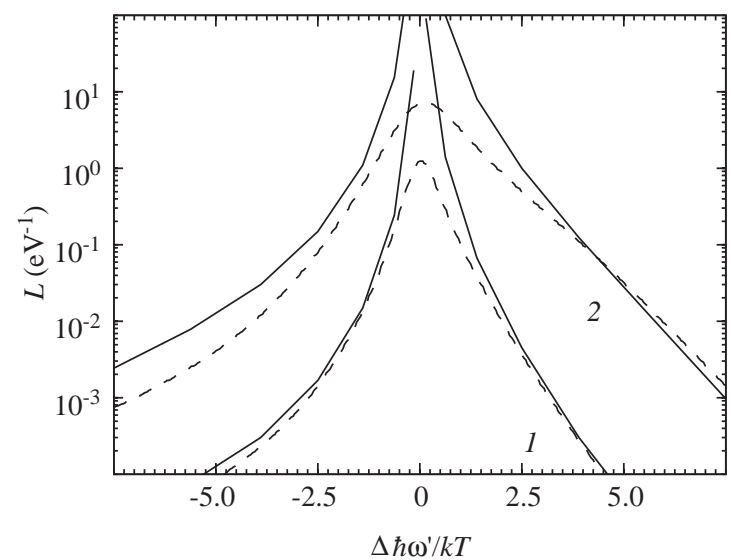

Fig. 2. Spectral dependence of the emission line at different relative excitation levels (1) $\Delta F-E_{\mathrm{g}}=-3 k T$ and (2) $\Delta F-$ $E_{\mathrm{g}}=3 k T$ at $T=286 \mathrm{~K}$. Solid and dotted lines give the results obtained by numerical integration and interpolation, correspondingly.

zero momentum. Such a replacement ensures the limiting transformation of resulting line (25) back to expression (23) at decrease of the excitation level. As can be seen in Fig. 2, the approximations fit the numerical data well in the line tails when the emission intensity decreases by more than order of magnitude.

\section{Discussion}

The results of calculations of the homogeneous broadening form-factor for different temperatures are presented in Fig. 3. We may conclude the following:

(1) The emission line shape function has asymmetric exponentially decaying tails. The longwavelength side (at the deep tails) decays slower than the short-wavelength one. The asymmetric exponential behavior of the decay is related to the statistical energy distribution of current carriers and the fact that the laws of conservation of energy and momentum are satisfied upon interaction. Such a behavior is not related to the form of the interaction matrix element. Therefore, a similar exponential factor is present in the line 


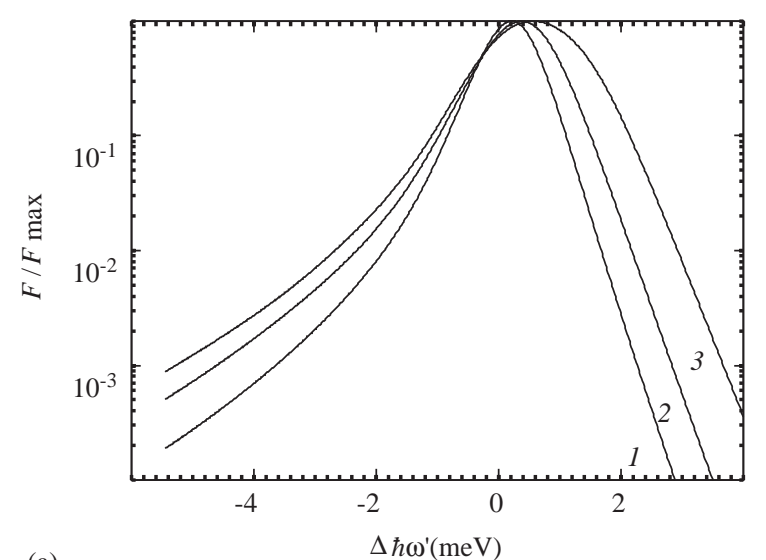

(a)

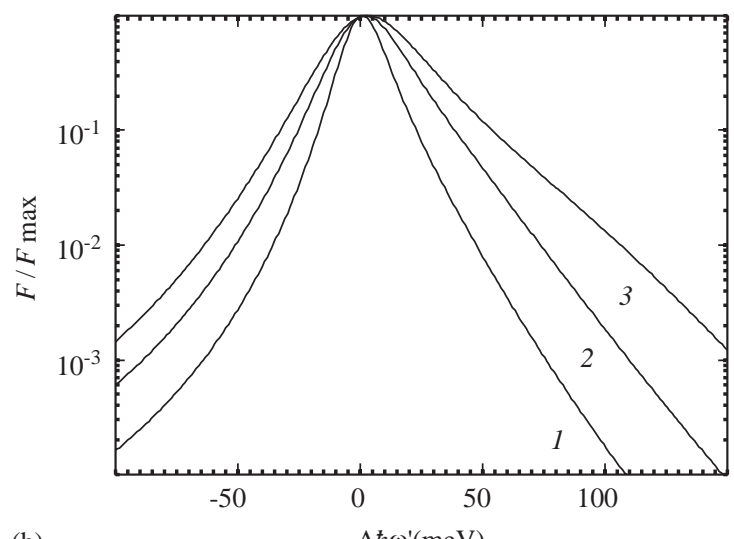

(b)

$\Delta \hbar \omega^{\prime}(\mathrm{meV})$

Fig. 3. Normalized on a peak value emission lines at temperatures (a) 4.2 and (b) $286 \mathrm{~K}$ and at various excitation levels (1) $\Delta F-E_{\mathrm{g}}=-3 k T$, (2) $\Delta F-E_{\mathrm{g}}=0$, and (3) $\Delta F-E_{\mathrm{g}}=3 k T$.

shape functions for quantum wires and bulk semiconductors.

(2) The emission line broadens as the excitation level increases. Mathematically, the broadening of an emission line is expressed by the fact that the integrated-emission intensity increases proportionally to $\exp (\Delta F / k T)$, while the emission intensity in the line tails is $\exp (3 \Delta F / 2 k T)$. Taking into account the fact that the growth in the excitation level leads to an increase in the number of particles in the bands, the line broadening is explained as follows: the increase in the rate of the three-particle processes (which is proportional to the third power of the number of particles $n^{3}$ ) anticipates the increase in the rate of direct radiative transitions involving two particles (which is proportional to $n^{2}$ ).

(3) Asymmetry of the emission line is more essential at low temperatures, where equality of the range of many-body energy level splitting $\gamma$ and thermal energy $k T$ is realized at a smaller relative excitation level $(\Delta F-$ $\left.E_{\mathrm{g}}\right) / k T$ (see expression (24)). Therefore the shape of the spectral line is greatly influenced by the statistical factor (see Fig. 3a).

The qualitative features of the emission line listed above are in accordance with results of experimental works [3,2]. Although the quantitative comparison with the experiment requires more detailed consideration of the peculiarities of the energy-band structure, we have presented some data of spectral line widths and logarithmic decrements of the tails (Fig. 4) obtained within the framework of the parabolic band approximation used.

Values of the logarithmic decrement for the long-wavelength side of the spontaneous radiation spectrum in QWs of the GaInAs-GaAs heterosystem, obtained in work [3] at temperatures 4.2, 77 , and $286 \mathrm{~K}$ under high excitation, were accordingly made $3.3,6.9$, and $8.5 \mathrm{meV}$. Numerical calculations for the same conditions gave $0.9,6$, and $15 \mathrm{meV}$. The largest discrepancy is observed at the temperature of $4.2 \mathrm{~K}$.

However, if we take into account that the temperature of electron gas differs from the temperature of the crystal lattice and, as it has been estimated by authors [3] from damping the short-wavelength side of the spectrum, $22 \mathrm{~K}$, the theoretical logarithmic decrement of the longwavelength spectrum side would be about $2.4 \mathrm{meV}$ that is close enough to the experimental data. The observed weakness of the dependence of the logarithmic decrement on the current at small temperatures can be explained by the increase of the degeneracy factor of the electron-hole system $\left(\Delta F-E_{\mathrm{g}}\right) / k T$ at cooling under conditions of constant injection current.

In work [2] for QWs in the GaAs-AlGaAs heterosystem at room temperature, the measured logarithmic decrement for the long-wavelength 


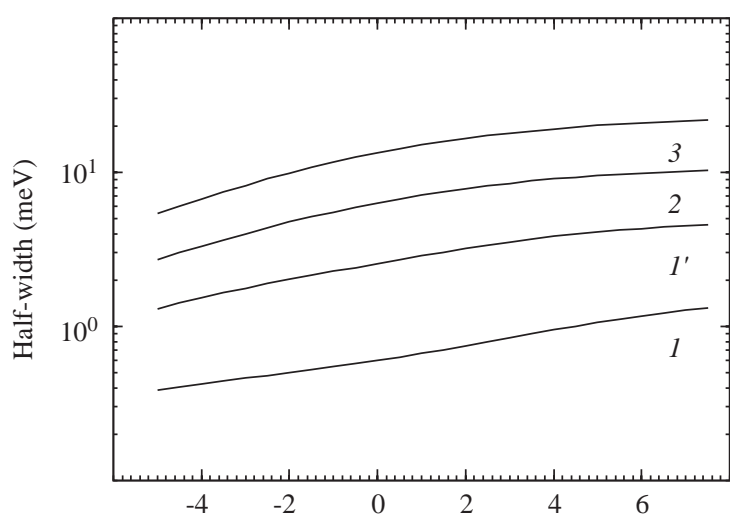

(a)

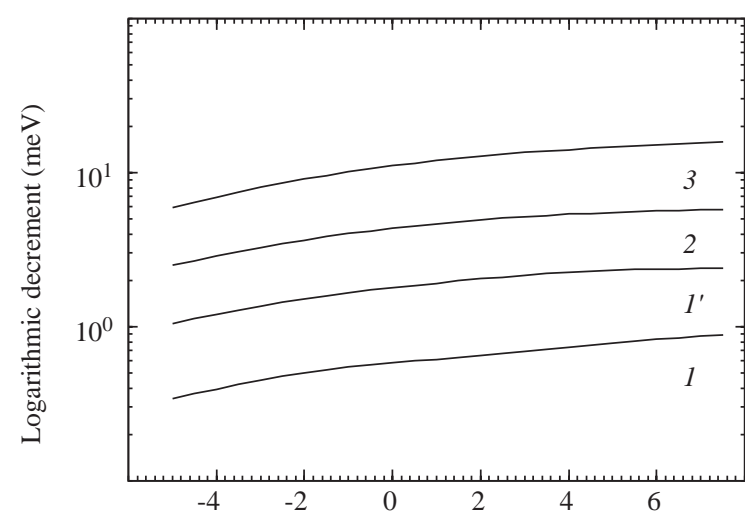

(b)

$\Delta F / k T$

Fig. 4. (a) Half-width of the emission line and (b) the logarithmic decrement at the long-wavelength side versus the excitation level normalized on the thermal energy for various temperatures (1) 4.2, (1') 22, (2) 77, and (3) $286 \mathrm{~K}$.

side of the spontaneous radiation spectrum changes from 9 to $12 \mathrm{meV}$ when variation of the carrier concentration is in the range of $10^{11}-10^{13} \mathrm{~cm}^{-2}$. Because of weak dependence of the spectral line on the carrier effective masses, it is also very close to the calculated data.

Note that here, the widths of spectral lines, estimated with the use of the intraband relaxation time of carriers, are quantitatively comparable to the presented results, but have an opposite dependence on the excitation level. As it was concluded in work [1], at the non-equilibrium carrier concentration of $10^{12} \mathrm{~cm}^{-2}$ the carrier intraband relaxation time increases and, therefore, the emission linewidth has to decrease. The former feature has not been observed experimentally $[3,2,17]$. In this case, the probability of optical transitions is nearly equal in a wide energy range and in the central part of the spectrum the optical transitions can be considered as recombination with no $\mathbf{k}$-selection rule [18].

The effects of electron-phonon interaction, that also result in line broadening [1,4,15], are not included here. It is possible to extend the present theory and find the phonon-related components in addition to (19)-(22). For example, consideration of carrier-LO phonon interaction will result in a smooth line-shape function (not only in the deltalike sidebands) if phonon dispersion is taken into account.

\section{Conclusion}

The performed theoretical analysis of the emission line broadening at the Coulomb interaction of carriers has shown that (i) exponential decays are associated with statistics of current carriers energy distribution, (ii) asymmetry of the line originates from fulfilment of laws of conservation of energy and momentum in the electron-hole system with Coulomb interactions, and (iii) the emission-line broadening with an increase in the excitation level is explained by the fact that the increase in the rate of the three-particle process, which determines the intensity of the line tails, anticipates the rate of direct radiative two-particle transitions, which determine the intensity of the central part of the spectral line.

\section{References}

[1] M. Asada, IEEE J. Quantum Electron. 25 (1989) 2019.

[2] M.P. Kesler, Ch. Harder, IEEE J. Quantum Electron. 27 (1991) 1812.

[3] P.G. Eliseev, I.V. Akimova, Semiconductors 32 (1998) 423; P.G. Eliseev, I.V. Akimova, Semiconductors 32 (1998) 428.

[4] M. Yamanishi, Y. Lee, IEEE J. Quantum Electron. 23 (1987) 367.

[5] T. Ohtoshi, M. Yamanishi, IEEE J. Quantum Electron. 27 (1991) 46.

[6] A. Tomita, A. Suzuki, IEEE J. Quantum Electron. 27 (1991) 1630. 
[7] D. Ahn, IEEE J. Select. Top. Quantum Electron. 1 (1995) 301.

[8] M. Kira, F. Jahnke, W. Hoyer, S.W. Koch, Prog. Quantum Electron. 23 (1999) 189.

[9] S.W. Koch, T. Meier, F. Jahnke, P. Thomas, Appl. Phys. A 71 (2000) 511.

[10] S.W. Koch, T. Meier, W. Hoyer, M. Kira, Physica E 14 (2002) 45.

[11] K. Hannewald, S. Glutsch, F. Bechstedt, Phys. Rev. B 62 (2000) 4519.

[12] K. Hannewald, S. Glutsch, F. Bechstedt, Phys. Rev. B 61 (2000) 10792.
[13] K. Hannewald, S. Glutsch, F. Bechstedt, Phys. Rev. Lett. 86 (2001) 2451.

[14] A.A. Afonenko, Semiconductors 38 (2004) 335.

[15] J. Foörstner, K.J. Ahn, J. Danckwerts, M. Schaarschmidt, I. Waldmuüller, C. Weber, A. Knorr, Phys. Stat. Sol. (b) 234 (2002) 155.

[16] A.A. Afonenko, J. Appl. Spectrosc. 71 (2004) 237.

[17] V.D. Kurnosov, K.V. Kurnosov, R.V. Chernov, Quantum Electron. 32 (2002) 303.

[18] A.A. Afonenko, I.S. Manak, V.A. Shevtsov, V.K. Kononenko, Semiconductors 31 (1997) 929. 